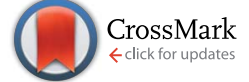

Cite this: RSC Adv., 2016, 6, 89380

Received 1st July 2016

Accepted 6th September 2016

DOI: $10.1039 / c 6 r a 16935 j$

www.rsc.org/advances

\section{Spatial control of direct chemical vapor deposition of graphene on silicon dioxide by directional copper dewetting $\dagger$}

\author{
Wesley T. E. van den Beld, ${ }^{\star}$ Albert van den Berg and Jan C. T. Eijkel
}

\begin{abstract}
In this paper we present a method for the spatial control of direct graphene synthesis onto silicon dioxide by controlled dewetting. The dewetting process is controlled through a combination of using a grooved substrate and conducting copper deposition at an angle. The substrate is then treated using a typical graphene chemical vapor deposition synthesis process at an elevated temperature during which directional dewetting of the copper into the grooves occurs while graphene is deposited at the mesas in between the grooves. The dewetting process and the synthesized graphene layer are characterized. The method is a non-manual, controllable and wafer-scale process, and therefore opens new possibilities for the construction of functional devices such as e.g. transistors.
\end{abstract}

Since its discovery in 2004 as a functional material, there has been consistent growth in interest for graphene, owing to its unique electronic and mechanical properties. ${ }^{1-5}$ For most practical devices, such as transistors, it is essential to be able to synthesize a single layer of graphene on a dielectric surface. ${ }^{\mathbf{4}, 6,7}$ The first method successfully used to isolate a single layer of graphene was exfoliation of highly ordered pyrolytic graphite (HOPG) on silicon dioxide. ${ }^{8}$ Although this method results in high quality graphene, it is labor intensive, offers only a low yield and is therefore not useful for mass production. Another route to isolate graphene is by dissolving graphite in an oxidizing solution in combination with a drying procedure. ${ }^{9-11}$ Such chemical methods typically result in a large spread in the number of graphene layers. ${ }^{12}$ As an alternative, chemical vapor deposition (CVD) processes enable large area single layer graphene production. Usually, transition metals (copper, nickel, etc.) are used as catalysts for the decomposition of gaseous alkanes (methane, ethane). ${ }^{13}$ To release the CVD graphene from the metal substrate however, a manual transfer protocol is required, which makes the process again labor intensive and hard to automate. Therefore a direct synthesis process on dielectric substrates is seen to be crucial for the future of integration of graphene into practical devices. Metal-free graphene synthesis directly on insulators has also been shown to be possible, however these metal-free methods are typically

BIOS - Lab on a Chip Group, MESA ${ }^{+}$Institute for Nanotechnology, MIRA Institute for Biomedical Engineering and Technical Medicine, University of Twente, The Netherlands. E-mail: w.t.e.vandenbeld@utwente.nl; Tel: +31534895653

$\dagger$ Electronic supplementary information (ESI) available: Optical microscopy data of dewetted mesas, Raman spectroscopy analysis results of deposited graphene, atomic force microscopy data of ridges on mesas, energy selective backscatter data of copper nanoparticles and CVD log data. See DOI: 10.1039/c6ra16935j accompanied by very long synthesis times caused by very low reaction rates compared to methods that make use of copper and nickel catalysts. ${ }^{14-16}$ The feasibility of graphene synthesis on copper oxide where copper oxide is also the catalytic material has been demonstrated. ${ }^{17}$ However, the electrical properties of copper oxide make it unsuitable for application in devices. ${ }^{\mathbf{1 8}}$ Other research has shown that direct graphene synthesis is possible by dewetting of thin copper films on silica substrates using a CVD protocol. ${ }^{19}$ A disadvantage of this route is that dewetting copper on a flat substrate results in a randomly distributed patchy graphene pattern. ${ }^{19-23}$ Furthermore, the remaining copper particles will not fully evaporate, even after a long annealing time. ${ }^{19,21}$ When very long process times are used, the remaining copper particles can eventually even sink into the silica substrate. ${ }^{24}$

In this paper we report a solution for this random copper dewetting consisting of an improved method for direct and controlled graphene synthesis on silicon dioxide using controlled dewetting and evaporation of copper. The dewetting process is controlled by a combination of using a grooved substrate and conducting copper deposition at an angle. Areas for graphene deposition are defined as mesas in between grooves produced using a potassium hydroxide (KOH) wet etching process on a silicon substrate. Subsequently the wafer is oxidized to form a layer of silicon dioxide $\left(\mathrm{SiO}_{2}\right)$, and a layer of copper $(\mathrm{Cu})$ is deposited on the wafer. There are several advantages of using a grooved substrate over e.g., copper strips. The copper dewets in a single direction which is defined by the copper deposition angle, making it possible to deposit a single graphene line on the mesa. In addition, minimal copper oxidation occurs, since the last step prior to graphene synthesis is the deposition of copper enabling a proper catalytic surface 
for graphene deposition. In this research copper was chosen because of its excellent graphene synthesis catalytic properties, low carbon solubility and dewetting properties. ${ }^{19,22,25}$ For CVD graphene synthesis on thin copper films a lower temperature is required compared to copper foils. ${ }^{26}$ During the CVD process, graphene is continuously deposited directly onto the silicon dioxide mesa (the flat area between the grooves as displayed in Fig. 1a) while the copper is evaporating and dewetting into the silicon dioxide grooves. ${ }^{\mathbf{1 9 , 2 2}}$ The quality of the deposited graphene which stays directly on the silicon dioxide has been optimized by varying the partial pressures of the reactive gasses in the CVD process. The method does not require graphene transfer and therefore opens new ways for the implementation of automated wafer-scale graphene synthesis.

The design of the fabricated grooved substrate is shown in Fig. 1a. The mesa widths $m$ range from $1 \mu \mathrm{m}$ to $10 \mu \mathrm{m}$ and the groove width $w$ is set to $m / 0.4$. The groove angle $\alpha_{\mathrm{g}}$ is determined by the silicon $\langle 100\rangle$ crystal orientation as $54.7^{\circ}$. The silicon substrate was oxidized, resulting in a silicon dioxide layer of $300 \mathrm{~nm}$ serving as a copper diffusion barrier. Subsequently copper and graphene were deposited as schematically shown in Fig. 1. The copper is deposited on the substrate at an angle $\alpha_{\mathrm{Cu}}$ by e-beam evaporation. This results in a copper thickness on the mesa of $h=h_{0} \cos \left(\alpha_{\mathrm{Cu}}\right)$, where the deposition thickness $h_{0}$ is set to $500 \mathrm{~nm}$. Subsequently, the substrate is treated by a CVD process at a temperature of $1000{ }^{\circ} \mathrm{C}$. In this

a)

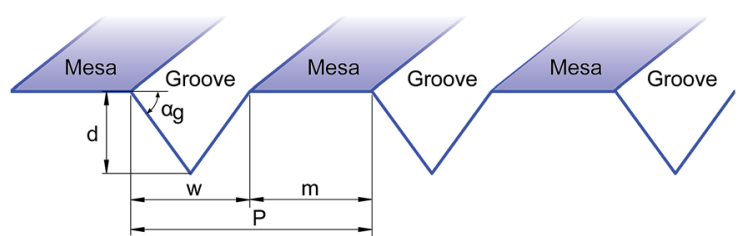

b)

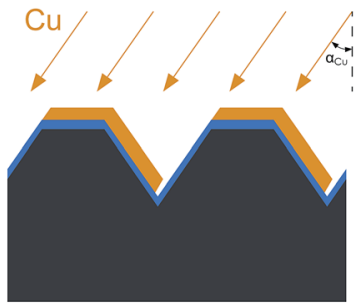

c)

d)
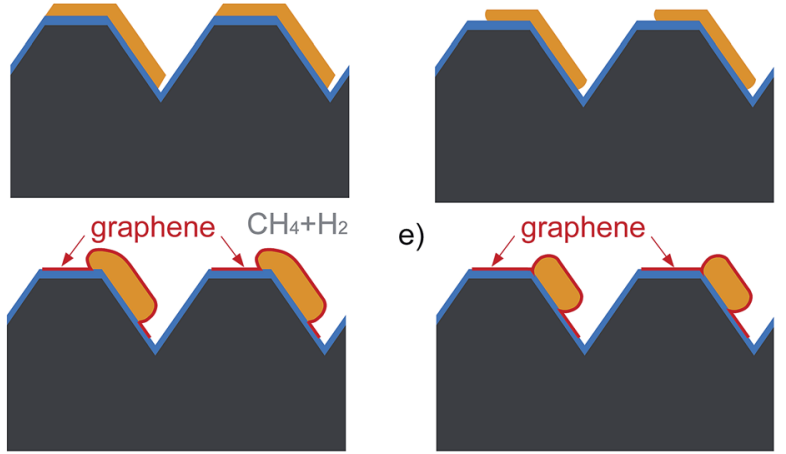

e)

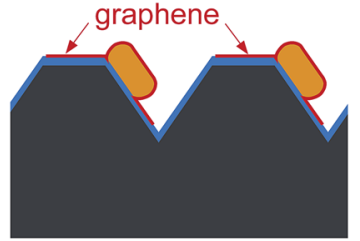

Fig. 1 Design of the substrate with groove width $w$ and mesa width $m$ which repeat in period $P(a)$. The groove is etched at an angle $\alpha_{\mathrm{g}}$ resulting in a groove depth $d$. Schematic of the method: copper deposition at an angle on the silicon dioxide/silicon substrate (b), conformation of the copper to the contact angle during heating (c), propagation of the contact line by dewetting and evaporation of the copper during the CVD process leaving a graphene layer (d) and the final situation with copper in the groove and the mesa covered by graphene (e) process the copper conforms initially to the copper silicon dioxide contact angle, followed by dewetting which is driven by the gradient of the curvature in the copper landscape. ${ }^{27,28}$ During this dewetting process, graphene is synthesized and left on the silicon dioxide surface by the retreating copper. ${ }^{1927-29}$ Three phenomena can be observed in the dewetting process: conformation to the contact angle, propagation of the contact line and occasional film punch-through.

Firstly, to minimize surface energy, a fast rearrangement at the edge of the film will occur in the initial stage until the contact angle $\theta_{c}$ is obtained. ${ }^{30}$ This contact angle of the dewetted copper with the surface can be calculated using Young's equation

$$
\gamma_{\mathrm{i}}+\gamma_{\mathrm{m}} \cos \theta_{\mathrm{c}}=\gamma_{\mathrm{s}}
$$

The interface energy $\gamma_{\mathrm{i}}$ at the copper-silicon dioxide interface is $1.1 \mathrm{~J} \mathrm{~m}^{-2}$, the surface energy of copper $\gamma_{\mathrm{m}}$ is $1.3 \mathrm{~J} \mathrm{~m}^{-2}$ and the silicon dioxide surface energy $\gamma_{\mathrm{s}}$ is about $0.3 \mathrm{~J} \mathrm{~m}^{-2}$ close to the melting temperature of copper $\left(1083{ }^{\circ} \mathrm{C}\right)$, resulting in a contact angle of approximately $128^{\circ} . .^{31,32}$

Secondly, after the fast initial conformation, copper evaporation and dewetting drive the propagation of the contact line..$^{30,33}$ The dewetting transport is dominated by surface diffusion of the copper (bulk transport can be neglected), minimizing the surface chemical potential and therefore smoothing the film over time..$^{30,34,35}$ The gradient of the curvature $K$ over the surface landscape $s$ leads to a metal flux $J_{\mathrm{m}}$ (assuming an isotropic surface energy) according to: ${ }^{28}$

$$
J_{\mathrm{m}}=\frac{-D_{\mathrm{s}} \gamma_{\mathrm{m}} N_{\mathrm{s}} \Omega}{k T} \frac{\partial K}{\partial s}
$$

where $N_{\mathrm{s}}$ is the number of copper atoms per unit area, $\Omega$ is the molecular volume of copper and $k T$ is the product of the Boltzmann constant and the temperature. The surface diffusion constant $D_{\mathrm{s}}$ is strongly temperature dependent as $D_{\mathrm{s}}=D_{0} \mathrm{e}^{\frac{-Q_{\mathrm{s}}}{k T N_{\mathrm{a}}}}$ where $N_{\mathrm{a}}$ is Avogadro's constant. For copper in a hydrogen environment at $1.0 \mathrm{mbar}$ the surface diffusion pre-exponential is $D_{0}=1 \times 10^{-5} \mathrm{~m}^{2} \mathrm{~s}^{-1}$ and the activation energy is $Q_{\mathrm{s}}=92$ $\mathrm{kJ} \mathrm{mol}{ }^{-1} \cdot{ }^{36}$ The temperature dependence of this surface diffusion allows tuning of the retraction velocity of the contact line. For metal films on flat substrates, the contact line will propagate as a function of time in the form of $x_{\mathrm{cl}} \sim t^{\alpha_{\mathrm{dw}}}$, where $\alpha_{\mathrm{dw}}$ is dependent on geometrical film assumptions. ${ }^{37}$ In most studies an $\alpha_{\mathrm{dw}}$-factor of about $\frac{2}{5}$ is found, which is practically independent of the contact angle..$^{30,37,38}$ The mechanism for graphene deposition proposed by Ismach et al. ${ }^{\mathbf{1 9}}$ is that graphene is synthesized by the catalytic action of the copper. While the contact line is retracting, graphene is transferred from the copper to the silicon dioxide surface, resulting in an insulating surface covered by graphene.

The deposited copper film is conformal and will have a constant curvature at the mesa-groove corner prior to dewetting (see Fig. 2a). To reduce the overall curvature of the copper surface, a dewetting flux will drive copper away from the mesagroove corner as follows from eqn (2), resulting in local 

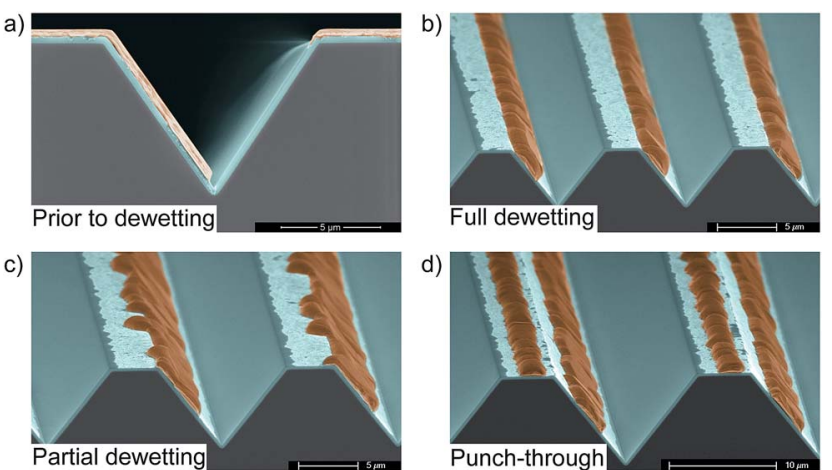

Fig. 2 False colored SEM images of a cross section of the grooved substrate with $300 \mathrm{~nm}$ of silicon dioxide (blue) with $500 \mathrm{~nm}$ of copper (orange) deposited at $35^{\circ}$ prior to (a) and after the chemical vapor process showing full dewetting (b), partial dewetting (c) and punchthrough (d) with mesa widths of $3.0 \mu \mathrm{m}, 4.1 \mu \mathrm{m}$ and $5.0 \mu \mathrm{m}$, respectively.

flattening of the surface. ${ }^{34,39}$ This process leads to a thinning of the copper film, eventually leading to a punch-through of the copper film by the mesa-groove corner.

To determine the copper-silicon dioxide contact angle, a copper film of $500 \mathrm{~nm}$ was deposited on a flat substrate and processed using CVD at $1000{ }^{\circ} \mathrm{C}$. Using scanning electron microscopy (SEM) a contact angle of $131^{\circ} \pm 4^{\circ}$ was measured, which is in agreement with the theoretical contact angle.

Subsequently, controlled dewetting on a grooved substrate was tested by depositing copper and applying the graphene CVD protocol. Three regimes could be distinguished in the resulting structures: fully dewetted, partially dewetted and punchedthrough, as can be observed in Fig. 2. In the case of full dewetting, the copper has retracted into the groove. On the mesa (and in the groove) a layer of graphene was found to be deposited. Wider mesas became partially dewetted, with fingers of copper still remaining on the mesa. For even wider mesas punch-through of the copper film at the mesa-groove corner occurs, resulting in a roll of copper remaining on the mesa.

The effect of a wafer cleaning procedure prior to copper deposition was investigated by testing the following procedures: 5 min UV-ozone, 5 min oxygen plasma, directly from an oxidation oven and no pre-clean. No significant influence from this pre-cleaning protocol on the dewetted mesa area was found.

To further control directional dewetting, copper was deposited at an angle on the grooved substrate. A series of deposition angles $\alpha_{\mathrm{Cu}}$ were tested to investigate their effect on the dewetted mesa area (see the ESI $\dagger$ for optical microscopy data). By image analysis of the dewetted surface, the relative dewetted mesa areas as a function of the mesa width and deposition angle were measured as shown in Fig. 3a. To the measurement data a logistic function was fitted in the form of

$$
f(m)=L\left(1-\frac{1}{1+\exp \left(-k\left(m-m_{\text {mid }}\right)\right)}\right)
$$

where $L, k$ and $m_{\text {mid }}$ are fitting parameters. The fit parameter $m_{\text {mid }}$, the midpoint of the logistic curve, as a function of
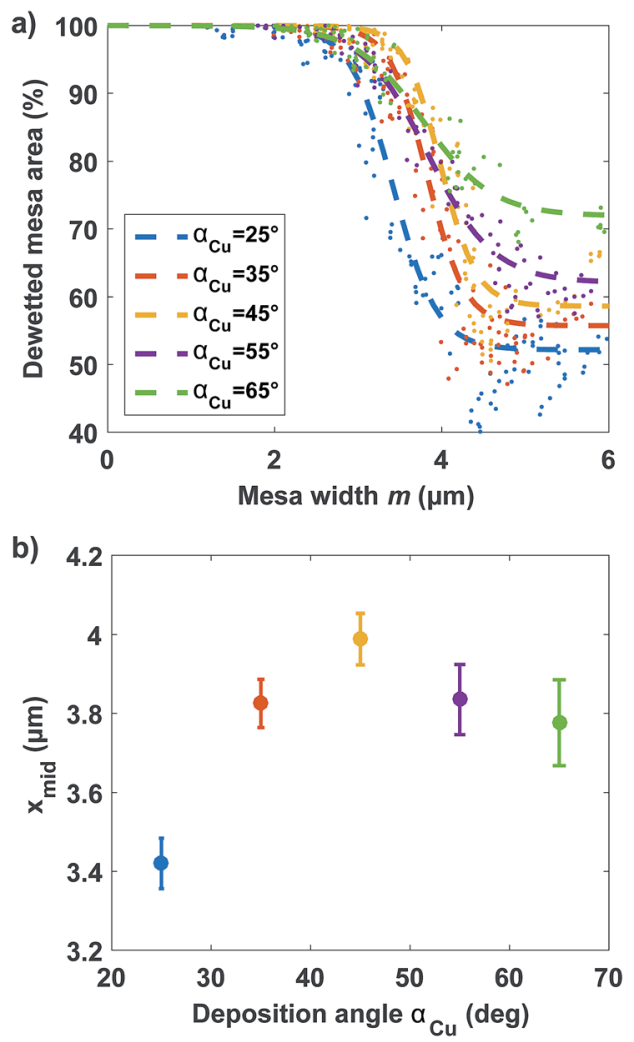

Fig. 3 The resulting dewetted mesa areas as a function of the total mesa width $m$ for a series of deposition angles $\alpha_{\mathrm{Cu}}$ (a), where the copper deposition thickness $h_{0}$ is set to $500 \mathrm{~nm}$. To this measurement data a logistic function is fitted. A dewetted mesa area of $100 \%$ corresponds to a fully dewetted mesa. The fit variable $m_{\text {mid }}$ of the logistic fit function (see eqn (3)) versus the copper deposition angle (b), showing an optimal deposition angle of $45^{\circ}$, the error bars show $95 \%$ fit confidence bounds.

deposition angle $\alpha_{\mathrm{Cu}}$ is shown in Fig. 3b. Since the groove angle $\alpha_{\mathrm{g}}$ is $54.7^{\circ}$, the minimum deposition angle $\alpha_{\mathrm{Cu}}$ for directional dewetting is $35^{\circ}$. The thickness of the copper film at the mesagroove corner is increased by larger deposition angles $\alpha_{\mathrm{Cu}}$, delaying punch-through and allowing the full dewetting of wider mesas. However, if the deposition angle $\alpha_{\mathrm{Cu}}$ becomes too large, the mesa copper thickness $h$ decreases rapidly. This thinner mesa copper film, in turn, breaks up into small structures $^{27}$ (also see the ESI $\dagger$ ), resulting in a larger transition region. Using a deposition angle of $45^{\circ}$ the widest fully dewetted mesas were obtained. It is of interest to discuss the effect of a possible variation in the groove angle. A much larger groove angle $\alpha_{\mathrm{g}}$ (sharper corner) will result in a faster punch-through and thus will reduce the maximum achievable fully dewetted mesa width. On the other hand, a much smaller groove angle (blunt corner) will strongly reduce the ability for directional dewetting. The optimal angle can differ from $54.7^{\circ}$, but variation of this angle on silicon is difficult as it is created by the crystal plane orientation.

To optimize the quality of the deposited graphene, several methods were tested in which hydrogen and methane gas flows were varied. The total gas inflow was set to $800 \mathrm{sccm}$ using 
argon. The process pressure was set to $10 \mathrm{mbar}$ to prevent too fast an evaporation of copper. ${ }^{33}$ The Raman spectra of the deposited graphene were subsequently recorded using a $532 \mathrm{~nm}$ laser. The graphene spectrum shows three characteristic peaks: the D peak $\left(1350 \mathrm{~cm}^{-1}\right)$ which indicates defects and discontinuities (e.g. crystal boundaries) in the graphene crystal, the $\mathrm{G}$ peak $\left(1590 \mathrm{~cm}^{-1}\right)$ which probes the in-plane bond stretching mode and the 2D peak $\left(2700 \mathrm{~cm}^{-1}\right)$ which holds information regarding the stacking orders. ${ }^{\mathbf{4 0 , 4 1}}$ To analyze the measured Raman spectra, Lorentzian peaks were fitted to the mapped Raman scan. A sharp (low FWHM) and symmetric 2D peak indicates single layer graphene. ${ }^{40}$ Ideally no defects are present, thus the $D$ peak intensity when normalized to the $G$ peak intensity $\left(I_{\mathrm{D}} / I_{\mathrm{G}}\right)$ should be low. Fewer graphene layers result in a higher $\mathrm{G}$ peak position, ${ }^{\mathbf{4 1 , 4 2}}$ however doping will also increase this position. ${ }^{43}$

A series of methods for graphene deposition were tested and the Raman spectroscopy fitting results can be found in the ESI. $\dagger$ The four most significant graphene quality indicators displayed are indicators for the number of layers and the defect density (including grain size). In this process we aim to produce defect free, single layer graphene. When analyzing the graphene quality indicators discussed above, the method using $50 \mathrm{sccm}$ methane and $50 \mathrm{sccm}$ hydrogen was selected for subsequent graphene deposition experiments.
The spatial distribution of the graphene quality was analyzed and can be found in Fig. 4. Where the copper dewetting started, first no graphene was deposited. This is related to the high temperature required to synthesize graphene, which is not yet reached when the copper starts dewetting. From this point on, the presence of a continuous layer of graphene was confirmed using Raman spectroscopy. The first deposited graphene layer possesses a higher D peak compared to the rest of the mesa. We expect variation in the dewetting velocity to influence the quality of the deposited graphene. The dewetting velocity can be influenced by, for example, the process temperature. We therefore believe that it is possible to improve the graphene quality further by tuning the dewetting velocity. A more constant dewetting velocity is expected to result in a more uniform graphene layer. This could be achieved by an increase in the temperature or a decrease in the pressure during the deposition process. By slightly increasing the temperature during the process the dewetting velocity will also increase. Increasing the process pressure will reduce the evaporation rate of copper which would lead to an increase in the graphene domain size. Lastly, the quality of the deposited graphene could be improved by further optimization of the hydrogen, methane and argon gas flow in the synthesis method. The impact of decreasing the dewetting velocity is expected to be beneficial for the synthesis process. It could help in the synthesis of a continuous graphene layer with a lower defect density and an a)

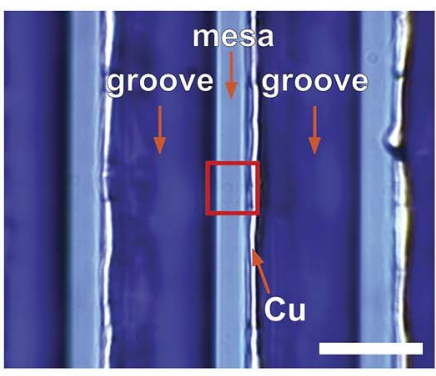

d)

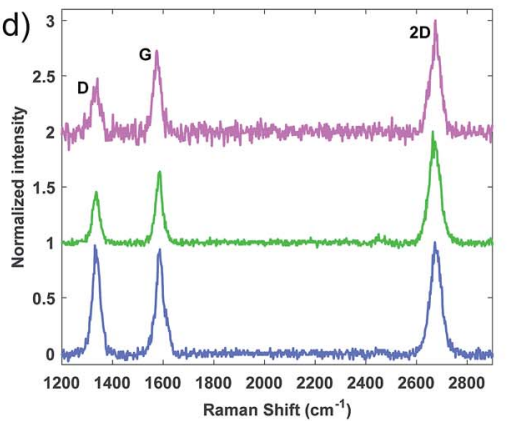

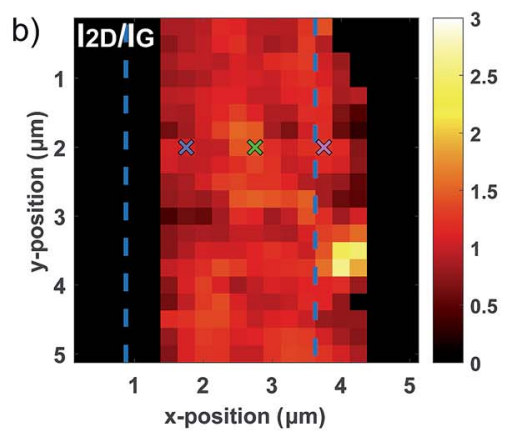

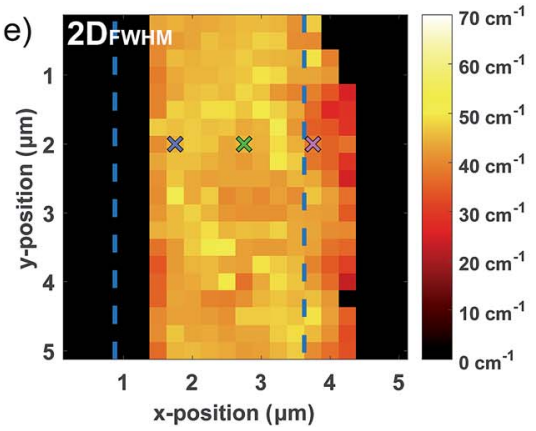

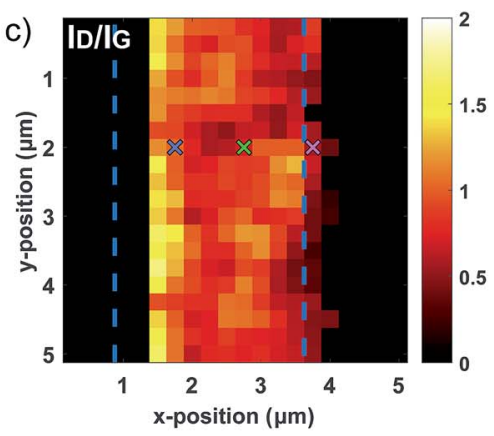

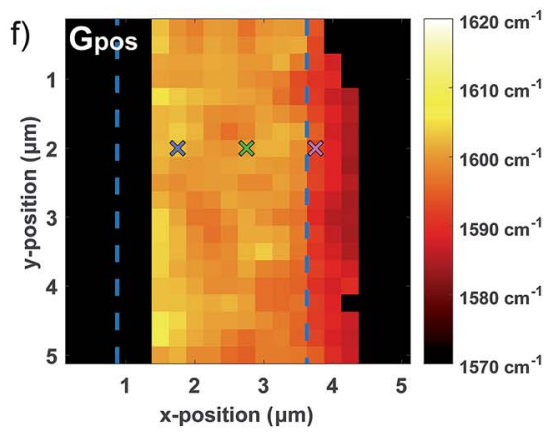

Fig. 4 Characterization of the deposited graphene on the mesa area, using the best CVD settings (hydrogen at 50 sccm and methane at 50 sccm). Raman spectra were recorded on the mesa (light blue) as indicated by the red box on the optical image, scale bar is $10 \mu \mathrm{m}$ (a). The colored crosses mark the locations of the displayed three Raman spectra (d). The dashed lines indicate the mesa edge in the mapped Raman scans, which reveal the graphene coverage on the mesa (b, $c, e, f)$. The apparent noise in the right most spectrum (pink) is caused by the copper next to the mesa (d). The $2 D$ to $G$ peak intensity $I_{2 D} / I_{G}$ shows uniform quality over the sample (b). The $D$ to $G$ peak intensity $I_{D} / I_{G}$ reveals more defects on the left edge of the mesa, where the copper dewetting had started (c). The 2D peak sharpness is uniform over the sample, however next to the mesa the 2D peak is sharper (e). The G peak position is higher on the mesa than next to the mesa (suspended and on copper), which is probably caused by the difference in the substrate induced doping (f). 
increased graphene domain size. Furthermore, less copper residues are expected at the expense of higher silicon oxide roughness.

After close inspection, SEM images revealed ridging in the silicon dioxide as can be seen in Fig. 5. By atomic force microscopy (AFM) the ridges were measured to have an average amplitude of $4 \mathrm{~nm}$ and a period of $47 \mathrm{~nm}$ (see the ESI $\dagger$ for AFM data). The forming of ridges during the copper dewetting process is explained by the transport of silicon dioxide along the interface with copper to the triple point where process gasses, copper, and silicon dioxide meet. The silicon dioxide is then transported by surface diffusion, which is inherent to metal ceramic systems at elevated temperatures. ${ }^{\mathbf{2 4 , 4 4 - 4 6}}$

In the study by Ismach et al. ${ }^{19}$ these ridges were also observed, however it was suggested that these ridges were wrinkles in the graphene. To confirm that the ridges in this work are in the silicon dioxide and not caused by e.g. graphene wrinkles, the sample was treated using oxygen plasma to strip the graphene. AFM images before and after this treatment showed a comparable roughness, from which we conclude that the silicon dioxide was ridged during the dewetting process. The continuity of the graphene layer was investigated using hydrofluoric acid (HF) treatment, since the graphene layer will protect the silicon dioxide against etching. After etching, the roughness was found to be comparable to the original sample, confirming that the graphene layer is continuous. In the AFM images nanoparticles of approximately $24 \mathrm{~nm}$ were observed on the sample surface. Analysis with an energy selective backscattered (EsB) detector and by energy-dispersive X-ray spectroscopy (EDX) showed that these nanoparticles are copper particles which must have been pinched off during the dewetting process (see the ESI $\uparrow$ for EsB data). The particles are most probably completely wrapped up in graphene. ${ }^{47}$ Interesting plasmonic properties have been reported in the literature, when these graphene coated copper particles spaced or have features in the order of tens of nanometers. ${ }^{\mathbf{4 8 - 5 0}}$ The graphene coating protected the copper from oxidation while it increased the sensitivity of measurements of analyte adsorption on the graphene. ${ }^{50-52}$ Removal of

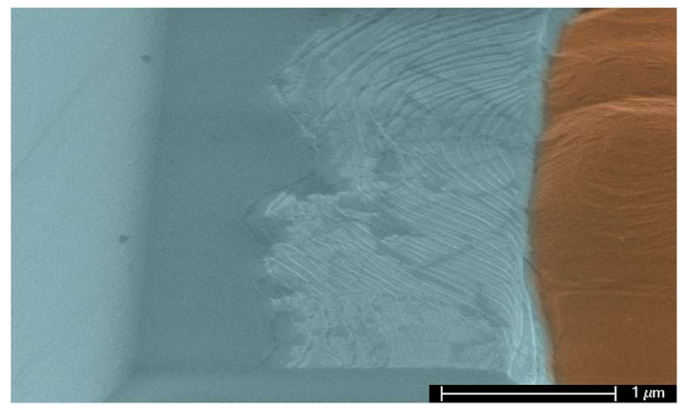

Fig. 5 False colored SEM image of the grooved substrate showing the fully dewetted mesa surface with silicon dioxide (blue) and the dewetted copper (orange). On the mesa surface, ridges and graphene are found which are both formed during copper dewetting in the CVD process. these copper residues would be possible by subsequent wet etching of the copper, since this removes the copper particles and leaves the graphene supported by the substrate, ${ }^{53}$ where the etchant is thought to reach the copper via the defects in the graphene.

The deposited graphene could be used as a transistor channel, which was demonstrated by the construction of a device in which the graphene can be gated. The synthesized graphene was interfaced with gold electrodes and a well was made with epoxy resin which served as a reservoir for an electrolyte solution. By solution gating a high gate capacitance is achieved, inducing high doping levels. ${ }^{43,54}$ In Fig. 6 the rectifying behavior of the graphene as a function of gating voltage is shown, which is normally not present in single layer graphene field effect transistor devices. ${ }^{55}$ The curve we found indicates the presence of a band gap in the graphene, ${ }^{56}$ which is useful for transistor devices.

In addition to this, the synthesized graphene could be exploited for other functional devices such as (bio)sensors, since the synthesized graphene layer consists of relatively small grains (nanocrystalline ${ }^{\mathbf{1 9}}$ ) and therefore possesses a relative high density of reactive grain boundaries. Another potential application for this graphene deposition method, after further optimization, would be the fabrication of graphene nanoribbons.

In summary, the presented method has improved the direct synthesis process of graphene on silicon dioxide by controlling the location of the dewetted areas. For this purpose copper was deposited at an angle on a grooved substrate. The copper dewetted into the grooves, leaving a layer of graphene on the flat part of the silicon dioxide surface. Optimization of the CVD process has been performed to obtain the best synthesized graphene sample possible. Furthermore analysis showed that the graphene layer is closed and the silicon dioxide surface was ridged after the dewetting process. This improved method opens new possibilities for wafer-scale graphene synthesis directly on insulating surfaces.

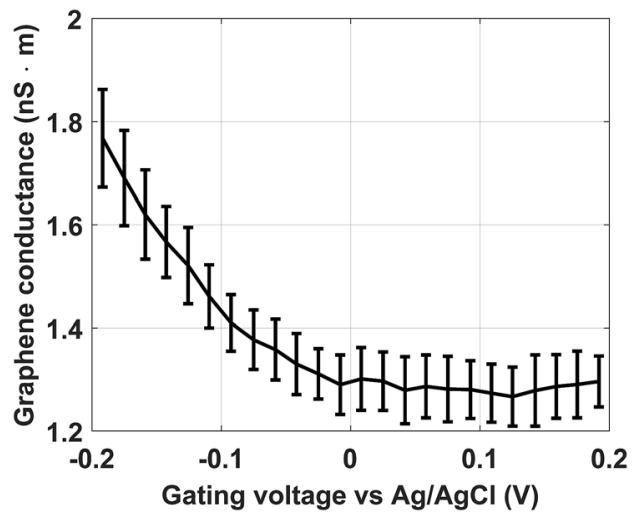

Fig. 6 The graphene in-plane conductance as a function of the gating voltage. The gating voltage was applied using a silver/silver chloride electrode in a $0.1 \mathrm{M}$ potassium chloride solution. The length of the error bars represent two standard deviation units of the measurement. 


\section{Experimental section}

An oxidized $\langle 100\rangle$ silicon wafer was patterned using conventional lithography with a line mask of 400 lines ranging from 1 $\mu \mathrm{m}$ to $10 \mu \mathrm{m}$ in width. After performing dry etching of the silicon dioxide, silicon was wet-etched anisotropically using a $25 \% \mathrm{KOH}$ solution at $75{ }^{\circ} \mathrm{C}$ to manufacture the grooves. Because of the crystal orientation of the wafer, a groove angle $\alpha_{\mathrm{g}}$ of $54.7^{\circ}$ is obtained. Subsequently, the grooved substrate was oxidized resulting in a silicon dioxide layer of $300 \mathrm{~nm}$ for optimal graphene visualization and to serve as a copper barrier at higher temperatures. Next, $500 \mathrm{~nm}$ of copper was deposited using e-beam evaporation at a controlled angle. Directly after this, the CVD process was performed in a cold-wall reaction chamber, which was purged with hydrogen prior to starting the process. Meanwhile, as the substrate was heated to $1000{ }^{\circ} \mathrm{C}$ (ramping up and down at $\sim 60^{\circ} \mathrm{C} \mathrm{min}^{-1}$ ), the CVD-process was executed at a pressure of 10 mbar with a hydrogen flow of 50 sccm, a methane flow of $50 \mathrm{sccm}$ and an argon flow of $700 \mathrm{sccm}$ for 15 minutes, see the ESI $\dagger$ for the CVD log data. The Raman spectra are recorded using a WITec alpha 300 system with a $532 \mathrm{~nm}$ laser at $1 \mathrm{~mW}$ using a $100 \times$ objective (0.9 NA) leading to a spot size of $\frac{1.22 \lambda}{\mathrm{NA}}=0.72 \mu \mathrm{m}$. For the fabrication of the graphene transistor device, gold contact pads were deposited by electron beam evaporation using a shadow mask, and were connected to a PCB by wire bonding. Subsequently, the gold pads and wire bonds were covered with epoxy (hysol), leaving the graphene area between the electrodes $(5 \mathrm{~mm})$ open. A droplet of $0.1 \mathrm{M} \mathrm{KCl}$ solution was applied to this area in which a silver/silver chloride electrode was inserted. Between the gold pads a voltage of $0.1 \mathrm{~V}$ was applied to measure the in-plane conductance. The graphene was solution gated by connecting the silver/silver chloride electrode to a Biologic SP300 potentiostat.

\section{Acknowledgements}

Financial support from Spinoza Grant for Albert van den Berg is acknowledged. This work was also supported by the Netherlands Center for Multiscale Catalytic Energy Conversion (MCEC), and an NWO Gravitation programme funded by the Ministry of Education, Culture and Science of the government of the Netherlands. Furthermore, the assistance of J. T. Loessberg-Zahl, H. Le The, J. G. M. Sanderink, J. W. Mertens and G. P. M. Roelofs is gratefully acknowledged.

\section{References}

1 M. I. Katsnelson, Mater. Today, 2007, 10, 20-27.

2 K. Geim and K. S. Novoselov, Nat. Mater., 2007, 6, 183-191.

3 K. Novoselov, Z. Jiang, Y. Zhang, S. Morozov, H. Stormer, U. Zeitler, J. Maan, G. Boebinger, P. Kim and A. Geim, Science, 2007, 315, 1379.

4 J. Bunch, S. Verbridge, J. Alden, A. Van Der Zande, J. Parpia, H. Craighead and P. McEuen, Nano Lett., 2008, 8, 2458-2462.
5 Y. Zhu, S. Murali, W. Cai, X. Li, J. Suk, J. Potts and R. Ruoff, Adv. Mater., 2010, 22, 3906-3924.

6 N. Lei, P. Li, W. Xue and J. Xu, Meas. Sci. Technol., 2011, 22, 107002.

7 F. Yan, M. Zhang and J. Li, Adv. Healthcare Mater., 2014, 3, 313-331.

8 K. S. Novoselov, A. K. Geim, S. Morozov, D. Jiang, Y. Zhang, S. Dubonos, I. Grigorieva and A. Firsov, Science, 2004, 306, 666-669.

9 V. C. Tung, M. J. Allen, Y. Yang and R. B. Kaner, Nat. Nanotechnol., 2009, 4, 25-29.

10 B. Cai, S. Wang, L. Huang, Y. Ning, Z. Zhang and G.-J. Zhang, ACS Nano, 2014, 8, 2632-2638.

11 S. Gilje, S. Han, M. Wang, K. L. Wang and R. B. Kaner, Nano Lett., 2007, 7, 3394-3398.

12 Z.-S. Wu, W. Ren, L. Gao, B. Liu, C. Jiang and H.-M. Cheng, Carbon, 2009, 47, 493-499.

13 R. Muñoz and C. Gómez-Aleixandre, Chem. Vap. Deposition, 2013, 19, 297-322.

14 J. Sun, T. Gao, X. Song, Y. Zhao, Y. Lin, H. Wang, D. Ma, Y. Chen, W. Xiang, J. Wang, et al., J. Am. Chem. Soc., 2014, 136, 6574-6577.

15 J. Sun, Y. Chen, M. K. Priydarshi, Z. Chen, A. Bachmatiuk, Z. Zou, Z. Chen, X. Song, Y. Gao, M. H. Rümmeli, et al., Nano Lett., 2015, 15, 5846-5854.

16 J. Chen, Y. Wen, Y. Guo, B. Wu, L. Huang, Y. Xue, D. Geng, D. Wang, G. Yu and Y. Liu, J. Am. Chem. Soc., 2011, 133, 17548-17551.

17 S. Gottardi, K. Müller, L. Bignardi, J. C. Moreno-López, T. A. Pham, O. Ivashenko, M. Yablonskikh, A. Barinov, J. Björk and P. Rudolf, Nano Lett., 2015, 15, 917-922.

18 A. Smith and H. Wieder, J. Phys. Chem., 1959, 63, 2013-2015. 19 A. Ismach, C. Druzgalski, S. Penwell, A. Schwartzberg, M. Zheng, A. Javey, J. Bokor and Y. Zhang, Nano Lett., 2010, 10, 1542-1548.

20 E. Jiran and C. Thompson, Thin Solid Films, 1992, 208, 23-28.

21 C. M. Müller and R. Spolenak, J. Appl. Phys., 2013, 113, 094301.

22 L. Croin, E. Vittone and G. Amato, Thin Solid Films, 2014, 573, 122-127.

23 T. Kaplas, D. Sharma and Y. Svirko, Carbon, 2012, 50, 15031509.

24 L. J. de Vreede, A. van den Berg and J. C. Eijkel, Nano Lett., 2015, 15, 727-731.

25 S. Bhaviripudi, X. Jia, M. S. Dresselhaus and J. Kong, Nano Lett., 2010, 10, 4128-4133.

26 L. Tao, J. Lee, H. Chou, M. Holt, R. S. Ruoff and D. Akinwande, ACS Nano, 2012, 6, 2319-2325.

27 C. V. Thompson, Annu. Rev. Mater. Res., 2012, 42, 399-434.

28 W. W. Mullins, J. Appl. Phys., 1957, 28, 333-339.

29 D. Wang, R. Ji and P. Schaaf, Beilstein J. Nanotechnol., 2011, 2, 318-326.

30 H. Wong, P. Voorhees, M. Miksis and S. Davis, Acta Mater., 2000, 48, 1719-1728.

31 C. J. Smithells and E. A. Brandes, Metals reference book, London etc, Butterworth, 1976.

32 J.-Y. Kwon, T.-S. Yoon, K.-B. Kim and S.-H. Min, J. Appl. Phys., 2003, 93, 3270-3278. 
33 G. Meng, T. Yanagida, M. Kanai, M. Suzuki, K. Nagashima, B. Xu, F. Zhuge, A. Klamchuen, Y. He and S. Rahong, Phys. Rev. E, 2013, 87, 012405.

34 A. Giermann and C. Thompson, J. Appl. Phys., 2011, 109, 083520.

35 D. Srolovitz and S. Safran, J. Appl. Phys., 1986, 60, 255-260.

36 F. Bradshaw, R. Brandon and C. Wheeler, Acta Metall., 1964, 12, 1057-1063.

37 F. Cheynis, F. Leroy and P. Müller, C. R. Phys., 2013, 14, 578589.

38 A. Presland, G. Price and D. Trimm, Prog. Surf. Sci., 1972, 3, 63-96.

39 W. W. Mullins, J. Appl. Phys., 1959, 30, 77-83.

40 A. Ferrari, J. Meyer, V. Scardaci, C. Casiraghi, M. Lazzeri, F. Mauri, S. Piscanec, D. Jiang, K. Novoselov and S. Roth, Phys. Rev. Lett., 2006, 97, 187401.

41 L. Malard, M. Pimenta, G. Dresselhaus and M. Dresselhaus, Phys. Rep., 2009, 473, 51-87.

42 A. C. Ferrari, Solid State Commun., 2007, 143, 47-57.

43 A. Das, S. Pisana, B. Chakraborty, S. Piscanec, S. Saha, U. Waghmare, K. Novoselov, H. Krishnamurthy, A. Geim and A. Ferrari, Nat. Nanotechnol., 2008, 3, 210-215.

44 E. Saiz, A. Tomsia and R. Cannon, Acta Mater., 1998, 46, 2349-2361.

45 E. Saiz, R. M. Cannon and A. P. Tomsia, Oil Gas Sci. Technol., 2001, 56, 89-96.
46 W. D. Kaplan, D. Chatain, P. Wynblatt and W. C. Carter, J. Mater. Sci., 2013, 48, 5681-5717.

47 M. Fuentes-Cabrera, B. H. Rhodes, J. D. Fowlkes, A. LópezBenzanilla, H. Terrones, M. L. Simpson and P. D. Rack, Phys. Rev. E, 2011, 83, 041603.

48 D. Ansell, I. Radko, Z. Han, F. Rodriguez, S. Bozhevolnyi and A. Grigorenko, Nat. commun., 2015, 6, 8846.

49 X. Li, X. Ren, Y. Zhang, W. C. Choy and B. Wei, Nanoscale, 2015, 7, 11291-11299.

50 X. Zhang, S. Xu, S. Jiang, J. Wang, J. Wei, S. Xu, S. Gao, H. Liu, H. Qiu, Z. Li, et al., Appl. Surf. Sci., 2015, 353, 63-70.

51 Y. Zhao, G. Chen, Y. Du, J. Xu, S. Wu, Y. Qu and Y. Zhu, Nanoscale, 2014, 6, 13754-13760.

52 V. Kravets, R. Jalil, Y.-J. Kim, D. Ansell, D. Aznakayeva, B. Thackray, L. Britnell, B. Belle, F. Withers, I. Radko, et al., Sci. Rep., 2014, 4, 5517.

53 C.-Y. Su, D. Fu, A.-Y. Lu, K.-K. Liu, Y. Xu, Z.-Y. Juang and L.-J. Li, Nanotechnology, 2011, 22, 185309.

54 J.-H. Zhong, J.-Y. Liu, Q. Li, M.-G. Li, Z.-C. Zeng, S. Hu, D.-Y. Wu, W. Cai and B. Ren, Electrochim. Acta, 2013, 110, 754-761.

55 T.-Y. Chen, P. T. K. Loan, C.-L. Hsu, Y.-H. Lee, J. Tse-Wei Wang, K.-H. Wei, C.-T. Lin and L.-J. Li, Biosens. Bioelectron., 2013, 41, 103-109.

56 J. Park, W. C. Mitchel, S. Elhamri, L. Grazulis, J. Hoelscher, K. Mahalingam, C. Hwang, S.-K. Mo and J. Lee, Nat. commun., 2015, 5677. 